\title{
Generalized Jensen's functional on time scales via extended Montgomery identity
}

Sofia Ramzan ${ }^{1 *} \mathbb{D}$, Ammara Nosheen ${ }^{1}$ (D), Rabia Bibi² and Josip Pečarić ${ }^{3}$

"Correspondence:

Ssofi227@gmail.com

'Department of Mathematics and Statistics, The University of Lahore (Sargodha Campus), Sargodha, Pakistan

Full list of author information is available at the end of the article

\begin{abstract}
In the paper, we use Jensen's inequality for diamond integrals and generalize it for $n$-convex functions with the help of an extended Montgomery identity. Moreover, the bounds have been suggested for identities associated with the generalized Jensen-type functional.
\end{abstract}

Keywords: Jensen's inequality; Convex functions; Diamond integrals; Time scales calculus

\section{Introduction and preliminaries}

Montgomery identity is well known in the literature. It is utilized to obtain a number of revolutionary inequalities such as trapezoid inequality, Ostrowski-type inequality, Čebyšev inequality, Grüss inequality, and Mohajani inequality. Mitrinović et al. in [17] proved that if $\chi:\left[b_{1}, b_{2}\right] \rightarrow \mathbb{R}$ is differentiable on $\left[b_{1}, b_{2}\right]$ and $\chi^{\prime}:\left[b_{1}, b_{2}\right] \rightarrow \mathbb{R}$ is integrable on $\left[b_{1}, b_{2}\right]$, then the Montgomery identity is

$$
\chi(t)=\frac{1}{b_{2}-b_{1}} \int_{b_{1}}^{b_{2}} \chi(p) d p+\int_{b_{1}}^{b_{2}} \mathfrak{P}(t, p) \chi^{\prime}(p) d p
$$

where

$$
\mathfrak{P}(t, p)= \begin{cases}\frac{p-b_{1}}{b_{2}-b_{1}}, & b_{1} \leq p \leq t, \\ \frac{p-b_{2}}{b_{2}-b_{1}}, & t<p \leq b_{2} .\end{cases}
$$

Bohner et al. in [7] proved the Montgomery identity on time scales and discussed it for discrete, continuous, and quantum cases. In case of $\mathbb{T}=\mathbb{R}$, it becomes (1).

Suppose that a probability density function $\psi:\left[b_{1}, b_{2}\right] \rightarrow[0, \infty)$, i.e., an integrable function which satisfies $\int_{b_{1}}^{b_{2}} \psi(p) d p=1$. Also $\xi(p)=\int_{b_{1}}^{p} \psi(t) d t$ when $p \in\left[b_{1}, b_{2}\right], \xi(p)=0$ when $p<b_{1}$ and $\xi(p)=1$ when $p>b_{2}$. Then the weighted generalization [19] of the Montgomery identity is

$$
\chi(t)=\int_{b_{1}}^{b_{2}} \psi(p) \chi(p) d p+\int_{b_{1}}^{b_{2}} \mathfrak{P}_{\psi}(t, p) \chi^{\prime}(p) d p,
$$

(c) The Author(s) 2021. This article is licensed under a Creative Commons Attribution 4.0 International License, which permits use sharing, adaptation, distribution and reproduction in any medium or format, as long as you give appropriate credit to the original author(s) and the source, provide a link to the Creative Commons licence, and indicate if changes were made. The images or other third party material in this article are included in the article's Creative Commons licence, unless indicated otherwise in a credit line to the material. If material is not included in the article's Creative Commons licence and your intended use is not permitted by statutory regulation or exceeds the permitted use, you will need to obtain permission directly from the copyright holder. To view a copy of this licence, visit http://creativecommons.org/licenses/by/4.0/. 
where the weighted Peano kernel is

$$
\mathfrak{P}_{\psi}(t, p)= \begin{cases}\xi(p), & b_{1} \leq p \leq t, \\ \xi(p)-1, & t<p \leq b_{2} .\end{cases}
$$

Sarikaya et al. [20] proved the weighted Montgomery identity on time scales.

To obtain our main results, we use the extended Montgomery identities by means of Taylor's formula I and II given in $[1,2]$.

Theorem 1 Let $E$ be an open interval in $\mathbb{R}$ and $b_{1}, b_{2} \in E$, s.t., $b_{1}<b_{2}$. Assume that a function $\chi: E \rightarrow \mathbb{R}$, s.t., $\chi^{(n-1)}$ is absolutely continuous for $n \in \mathbb{N}$, then

$$
\begin{aligned}
\chi(t)= & \frac{1}{b_{2}-b_{1}} \int_{b_{1}}^{b_{2}} \chi(p) d p+\sum_{\mathfrak{z}=0}^{n-2} \frac{\chi^{(\mathfrak{z}+1)}\left(b_{1}\right)}{\mathfrak{z} !(\mathfrak{z}+2)} \frac{\left(t-b_{1}\right)^{\mathfrak{z}+2}}{b_{2}-b_{1}} \\
& -\sum_{\mathfrak{z}=0}^{n-2} \frac{\chi^{(\mathfrak{z}+1)}\left(b_{2}\right)}{\mathfrak{z} !(\mathfrak{z}+2)} \frac{\left(t-b_{2}\right)^{\mathfrak{z}+2}}{b_{2}-b_{1}}+\frac{1}{(n-1) !} \int_{b_{1}}^{b_{2}} R_{n}(t, s) \chi^{(n)}(s) d s,
\end{aligned}
$$

where

$$
R_{n}(t, s)= \begin{cases}\frac{-(t-s)^{n}}{n\left(b_{2}-b_{1}\right)}+\frac{t-b_{1}}{b_{2}-b_{1}}(t-s)^{n-1}, & b_{1} \leq s \leq t, \\ \frac{-(t-s)^{n}}{n\left(b_{2}-b_{1}\right)}+\frac{t-b_{2}}{b_{2}-b_{1}}(t-s)^{n-1}, & t<s \leq b_{2} .\end{cases}
$$

Theorem 2 Let $E$ be an open interval in $\mathbb{R}$ and $b_{1}, b_{2} \in E$, s.t., $b_{1}<b_{2}$. Assume that a function $\chi: E \rightarrow \mathbb{R}$, s.t., $\chi^{(n-1)}$ is absolutely continuous for $n \in \mathbb{N}$, then

$$
\begin{aligned}
\chi(t)= & \frac{1}{b_{2}-b_{1}} \int_{b_{1}}^{b_{2}} \chi(t) d t+\sum_{\mathfrak{z}=0}^{n-2} \chi^{(\mathfrak{z}+1)}(t) \frac{\left(b_{1}-t\right)^{\mathfrak{j}+2}+\left(b_{2}-t\right)^{\mathfrak{z}+2}}{(\mathfrak{z}+2) !\left(b_{2}-b_{1}\right)} \\
& +\frac{1}{(n-1) !} \int_{b_{1}}^{b_{2}} \hat{R}_{n}(t, s) \chi^{(n)}(s) d s,
\end{aligned}
$$

where

$$
\hat{R}_{n}(t, s)= \begin{cases}\frac{-1}{n\left(b_{2}-b_{1}\right)}\left(b_{1}-s\right)^{n}, & b_{1} \leq s \leq t, \\ \frac{-1}{n\left(b_{2}-b_{1}\right)}\left(b_{2}-s\right)^{n}, & t<s \leq b_{2} .\end{cases}
$$

For $n=1, \sum_{\mathfrak{z}=0}^{n-2} \cdots$ is empty, therefore (5) and (7) reduce to Montgomery identity (1).

\subsection{On convex functions}

Suppose that a function $\mu: E \rightarrow \mathbb{R}$, it is termed convex if $\forall y_{1}, y_{2} \in E$ and $\gamma \in[0,1]$, the inequality

$$
\mu\left(\gamma y_{1}+(1-\gamma) y_{2}\right) \leq \gamma \mu\left(y_{1}\right)+(1-\gamma) \mu\left(y_{2}\right)
$$

holds. If $y_{1} \neq y_{2}$ and $\gamma \in(0,1)$ in (9), then $\mu$ is strictly convex. Moreover, $\mu$ is called concave function if (9) is reversed and inequality (9) is strictly concave if $\forall y_{1} \neq y_{2}$ and $\gamma \in(0,1)$ [19]. 
Divided differences are truly assigned to Newton, and Augustus de Morgan in 1842 used the term divided difference. The divided differences are beneficial if the functions have different degrees of smoothness. Divided differences are discussed in [13].

Definition 1 Suppose $\mu:[p, q] \rightarrow \mathbb{R}, n \in N$ and mutually distinct points $\eta_{0}, \eta_{1}, \ldots, \eta_{n}$ of $[p, q]$. Then the $n$th order divided difference of the function $\mu$ is

$$
\begin{aligned}
& {\left[\eta_{i} ; \mu\right]=\mu\left(\eta_{i}\right), \quad i=0, \ldots, n,} \\
& {\left[\eta_{0}, \ldots, \eta_{n} ; \mu\right]=\frac{\left[\eta_{1}, \ldots, \eta_{n} ; \mu\right]-\left[\eta_{0}, \ldots, \eta_{n-1} ; \mu\right]}{\eta_{n}-\eta_{0}} .}
\end{aligned}
$$

$n$-convex function is defined on the basis of $n$ th-order divided difference [19].

Suppose $n \geq 0$, then a function $\mu:[p, q] \rightarrow \mathbb{R}$ is termed $n$-convex iff $\forall(n+1)$ distinct points $y_{0}, y_{1}, \ldots, y_{n} \in[p, q]$,

$$
\left[y_{0}, y_{1}, \ldots, y_{n} ; \mu\right] \geq 0
$$

holds. The reverse effect of the above inequality implies that $\mu$ is $n$-concave. The strict effect of the above inequality implies that $\mu$ is a strictly $n$-convex (n-concave) function. The $n$ convexity of a function $\mu$ is examined by the following theorem [19].

Theorem 3 If $\mu^{n}$ exists, then $\mu$ is n-convex if and only if $\mu^{n} \geq 0$.

\subsection{On time scales}

In 1988, Hilger presented the notion of time scales calculus and proposed the unification of discrete and continuous time dynamical systems [14]. Gradually, this perspective of unification has been affixed by the extension and generalization characteristics.

$\Delta$ and $\nabla$ calculus is the initial approach to study time scales calculus. For detailed study of calculus on time scales, readers are reffered to $[6,8,9]$.

An arbitrary and nonempty closed subset of real numbers is called time scales $\mathbb{T}$. The real numbers $\mathbb{R}$ and the integers $\mathbb{Z}$ are most familiar examples of time scales.

Suppose $p \in \mathbb{T}$, then the mappings $\sigma(t), \rho(t): \mathbb{T} \rightarrow \mathbb{T}$ indicate the forward and backward jump operators respectively on time scale $\mathbb{T}$ and are defined as follows:

$$
\begin{aligned}
\sigma(t) & =\inf \{\mathfrak{e} \in \mathbb{T}: \mathfrak{e}>t\}, \\
\rho(t) & =\sup \{\mathfrak{e} \in \mathbb{T}: \mathfrak{e}<t\} .
\end{aligned}
$$

Take a point $t \in \mathbb{T}$, then $t$ is stated as follows:

- right-scattered, provided $\sigma(t)>t$;

- left-scattered, provided $\rho(t)<t$;

- isolated, provided $\rho(t)<t<\sigma(t)$;

- right-dense, provided $\sigma(t)=t$;

- left-dense, provided $\rho(t)=t$;

- dense, provided $\rho(t)=t=\sigma(t)$.

- The graininess functions $\sigma, v: \mathbb{T} \rightarrow[0,+\infty)$ are stated as

$$
\begin{gathered}
\sigma(t):=\sigma(t)-t, \\
\nu(t):=t-\rho(t) .
\end{gathered}
$$


Assume that a function $\mu: \mathbb{T} \rightarrow \mathbb{R}$, then it is termed:

- $r d$-continuous if it is continuous at all right-dense points in $\mathbb{T}$ and its left-sided limits are finite at all left-dense points in $\mathbb{T}$;

- ld-continuous if it is continuous at all left-dense points in $\mathbb{T}$ and its right-sided limits are finite at all right-dense points in $\mathbb{T}$.

We present the sets $\mathbb{T}^{k}, \mathbb{T}_{k}$, and $\mathbb{T}^{*}$ that originated from the time scale $\mathbb{T}$.

$\mathbb{T}^{k}=\mathbb{T}-t_{1}$, provided $\mathbb{T}$ contains a left-scattered maximum $t_{1} ;$ unless $\mathbb{T}^{k}=\mathbb{T}$.

$\mathbb{T}_{k}=\mathbb{T}-t_{2}$, provided $\mathbb{T}$ contains a right-scattered minimum $t_{2} ;$ unless $\mathbb{T}_{k}=\mathbb{T}$.

Lastly $\mathbb{T}^{*}=\mathbb{T}^{k} \cap \mathbb{T}_{k}$.

Suppose that a function $\mu: \mathbb{T} \rightarrow \mathbb{R}$ and $t \in \mathbb{T}^{k}$, then we describe $\mu^{\Delta}(t)$ (in case it exists) if, for given $\epsilon>0, \exists$ a neighborhood $W$ of $t$ so that $\forall y_{1} \in W$

$$
\left|\mu(\sigma(t))-\mu\left(y_{1}\right)-\mu^{\Delta}(t)\left[\sigma(t)-y_{1}\right]\right| \leq \epsilon\left|\sigma(t)-y_{1}\right| .
$$

Then $\mu$ is delta differentiable on $\mathbb{T}^{k}$ provided $\mu^{\Delta}(t)$ exists $\forall t \in \mathbb{T}^{k}$.

In the same way, for $t \in \mathbb{T}_{k}$, we say $\mu^{\nabla}(t)$ (in case it exists) if, for given $\epsilon>0, \exists$ a neighborhood $W$ of $t$ so that $\forall y_{1} \in W$

$$
\left|\mu(\rho(t))-\mu\left(y_{1}\right)-\mu^{\nabla}(t)\left[\rho(t)-y_{1}\right]\right| \leq \epsilon\left|\rho(t)-y_{1}\right| .
$$

Then $\mu$ is nabla differentiable on $\mathbb{T}_{k}$ provided $\mu^{\nabla}(t)$ exists $\forall t \in \mathbb{T}_{k}$.

Suppose that $\mu(t)$ is differentiable in the $\Delta$ and $\nabla$ sense on the time scales $\mathbb{T}$. Let $t \in \mathbb{T}$, $0 \leq \alpha \leq 1$, then $\diamond_{\alpha}$-dynamic derivative $\mu^{\diamond} \alpha(t)$ is

$$
\mu^{\diamond}(t)=\alpha \mu^{\Delta}(t)+(1-\alpha) \mu^{\nabla}(t) .
$$

Hence, $\mu$ is $\diamond_{\alpha}$-differentiable iff $\Delta$ and $\nabla$ derivatives of $\mu$ exist.

Remark 1 The $\diamond_{\alpha}$-derivative changes to

- $\Delta$ derivative, when $\alpha=1$;

- $\nabla$ derivative, when $\alpha=0$;

- "weighted dynamic derivative", when $\alpha \in(0,1)$.

For a complete advancement of the calculus on the $\diamond_{\alpha}$-derivative and $\diamond_{\alpha}$-integrals, refer to [21]. The refinement of $\diamond_{\alpha}$-derivative on time scales $\mathbb{T}$ is known as symmetric derivative given in [11], which is as follows:

Let $t \in \mathbb{T}_{k}^{k}$ and $\mu: \mathbb{T} \rightarrow \mathbb{R}$. Then $\mu^{\diamond}(t) \in \mathbb{R}$ (in case it exists) provided $\epsilon>0$, there is a neighborhood $V \subset \mathbb{T}$ of $t$, s.t.,

$$
\begin{aligned}
& \left|\left[\mu^{\sigma}(t)-\mu(y)+\mu(2 t-y)-\mu^{\rho}(t)\right]-\mu^{\diamond}(t)[\sigma(t)+2 t-2 y-\rho(t)]\right| \\
& \quad \leq \epsilon|\sigma(t)+2 t-2 y-\rho(t)|
\end{aligned}
$$

holds $\forall y \in V$ for which $2 t-y \in V$. 
Remark 2 The symmetric derivative is

- classic symmetric derivative, when $\mathbb{T}=\mathbb{R}$.

- symmetric difference operator, when $\mathbb{T}=h \mathbb{Z}, h>0$.

- q-symmetric difference operator, when $\mathbb{T}=q \mathbb{Z}, 0<q<1$.

A detailed discussion of symmetric derivatives on time scales is given in [11].

Suppose $\mu: \mathbb{T} \rightarrow \mathbb{R}$, then $U: \mathbb{T} \rightarrow \mathbb{R}$ is interpreted as delta antiderivative of $\mu$ if $U^{\Delta}(t)=$ $\mu(t)$ holds $\forall t \in \mathbb{T}^{k}$. The delta integral of $\mu$ is

$$
\int_{\ell_{1}}^{\ell_{2}} \mu(t) \Delta t=U\left(\ell_{2}\right)-U\left(\ell_{1}\right)
$$

Suppose $s: \mathbb{T} \rightarrow \mathbb{R}$, then $S: \mathbb{T} \rightarrow \mathbb{R}$ is interpreted as nabla antiderivative of $\mathrm{s}$ if $S^{\nabla}(t)=$ $s(t)$ holds $\forall t \in \mathbb{T}_{k}$. The nabla integral of $\mathrm{s}$ is

$$
\int_{\ell_{1}}^{\ell_{2}} s(t) \nabla t=S\left(\ell_{2}\right)-S\left(\ell_{1}\right)
$$

Suppose $\ell_{1}, t \in \mathbb{T}$ and $\hbar: \mathbb{T} \rightarrow \mathbb{R}$. Then $\diamond_{\alpha}$-integral is stated as

$$
\int_{\ell_{1}}^{t} \hbar(\mathfrak{s}) \diamond_{\alpha} \mathfrak{s}=\alpha \int_{\ell_{1}}^{t} \hbar(\mathfrak{s}) \Delta \mathfrak{s}+(1-\alpha) \int_{\ell_{1}}^{t} \hbar(\mathfrak{s}) \nabla \mathfrak{s},
$$

where $0 \leq \alpha \leq 1$. Since the diamond- $\alpha$ integral is a mixed form of $\Delta$ and $\nabla$ integrals, generally there does not exist

$$
\left(\int_{\ell_{1}}^{t} \hbar(\mathfrak{s}) \diamond_{\alpha} \mathfrak{s}\right)^{\diamond_{\alpha}}=\hbar(t), \quad t \in \mathbb{T} .
$$

In the special case, for each $t \in \mathbb{T}_{k}^{k}$, if a function is $\Delta$ and $\nabla$ differentiable at the same time, then $\mu$ is symmetric differentiable and

$$
\mu^{\diamond}(t)=\lambda(t) \mu^{\Delta}(t)+(1-\lambda(t)) \mu^{\nabla}(t)
$$

where

$$
\lambda(t)=\lim _{t \rightarrow y} \frac{\sigma(t)-y}{\sigma(t)+2 t-2 y-\rho(t)}
$$

is a real-valued function.

Remark 3 Clearly $0 \leq \lambda(t) \leq 1$ and

$$
\lambda(t)= \begin{cases}\frac{1}{2} & t \text { is dense } \\ \frac{\sigma(t)-t}{\sigma(t)-\rho(t)} & t \text { is not dense. }\end{cases}
$$

Suppose $\mu:\left[\ell_{1}, \ell_{2}\right]_{\mathbb{T}} \rightarrow \mathbb{R}, \ell_{1}<\ell_{2}$, and $\lambda(\cdot)$ is defined in (11). If $\lambda \mu$ is $\Delta$ integrable and $(1-\lambda) \mu$ is $\nabla$ integrable on $\left[\ell_{1}, \ell_{2}\right]_{\mathbb{T}}$, then $\diamond$-integral of $\mu$ on $\left[\ell_{1}, \ell_{2}\right]_{\mathbb{T}}$ is stated as

$$
\int_{\ell_{1}}^{\ell_{1}} \mu(t) \diamond t=\int_{\ell_{1}}^{\ell_{1}} \lambda(t) \mu(t) \Delta t+\int_{\ell_{1}}^{\ell_{1}}(1-\lambda(t)) \mu(t) \nabla t .
$$

More details on diamond integrals are given in [12]. 


\subsection{Jensen's inequality}

Inequality proved by Jensen [15] in 1906 is popular in mathematical analysis. For continuous and discrete analysis, it is used to formulate many classical inequalities. Therefore the developments in many other inequalities are based on the developments in Jensen's inequality. Jensen's inequality in a discrete version [15] is as follows:

Suppose that an interval $E$ in $\mathbb{R}$ and a convex function $\chi: E \rightarrow \mathbb{R}, v=\left(v_{1}, \ldots, v_{n}\right)$ is a real $n$-tuple and $y=\left(y_{1}, \ldots, y_{n}\right)$ is a positive $n$-tuple $(n \in \mathbb{N})$. Then

$$
\chi\left(\frac{\sum_{j=1}^{n} v_{j} y_{j}}{\sum_{j=1}^{n} v_{j}}\right) \leq \frac{\sum_{j=1}^{n} v_{j} \chi\left(y_{j}\right)}{\sum_{j=1}^{n} v_{j}} .
$$

The strict convexity of $\chi$ implies that (12) is strict unless $y_{1}=\cdots=y_{n}$ and $\chi$ is concave if (12) holds in reverse direction.

Jensen's inequality in an integral form [16] is as follows:

Suppose $b_{1}, b_{2} \in \mathbb{R}$ with $b_{1}<b_{2}$ and an interval $E$ in $\mathbb{R}$. If $\zeta \in C\left(\left[b_{1}, b_{2}\right], E\right)$ and a convex function $\chi \in C(E, \mathbb{R})$, then

$$
\chi\left(\frac{\int_{b_{1}}^{b_{2}} \zeta(p) d p}{b_{2}-b_{1}}\right) \leq \frac{\int_{b_{1}}^{b_{2}} \chi(\zeta(p)) d p}{b_{2}-b_{1}} .
$$

Anwar et al. in [4] extended the Jensen's inequality for $\Delta$-integrals as follows:

Suppose $\zeta \in C_{r d}\left(\left[b_{1}, b_{2}\right]_{\mathbb{T}}, E\right)$ and a convex function $\chi \in C(E, \mathbb{R})$ with $E \subset \mathbb{R}$. Let $b_{1}, b_{2} \in$ $\mathbb{T}$ with $b_{1}<b_{2}$ and $\hbar \in C_{r d}\left(\chi:\left[b_{1}, b_{2}\right]_{\mathbb{T}}, \mathbb{R}\right)$ satisfying $\int_{b_{1}}^{b_{2}}|\hbar(p)| \Delta p>0$, then

$$
\chi\left(\frac{\int_{b_{1}}^{b_{2}}|\hbar(p)| \zeta(p) \Delta p}{\int_{b_{1}}^{b_{2}}|\hbar(p)| \Delta p}\right) \leq \frac{\int_{b_{1}}^{b_{2}}|\hbar(p)| \chi(\zeta(p)) \Delta p}{\int_{b_{1}}^{b_{2}}|\hbar(p)| \Delta p} .
$$

Özkan et al. in [18] showed that if we apply nabla integrals instead of delta integrals, then (14) also holds. In [21], Sheng et al. presented $\diamond_{\alpha}$-dynamic derivative and $\diamond_{\alpha}$-dynamic integral for providing more balanced approximations with respect to computations.

Jensen's inequality for $\diamond_{\alpha}$-integral is given in [3].

Suppose $b_{1}, b_{2} \in \mathbb{T}$ with $b_{1}<b_{2}$ and $\alpha \in[0,1]$. Let $\hbar \in C\left(\left[b_{1}, b_{2}\right]_{\mathbb{T}}, \mathbb{R}\right)$ satisfying $\int_{b_{1}}^{b_{2}}|\hbar(p)| \diamond_{\alpha} p>0$. If $\zeta \in C\left(\chi:\left[b_{1}, b_{2}\right]_{\mathbb{T}}, E\right)$ and a convex function $\chi \in C(E, \mathbb{R})$ with an interval $E \subset \mathbb{R}$, then we have

$$
\chi\left(\frac{\int_{b_{1}}^{b_{2}}|\hbar(p)| \zeta(p) \diamond_{\alpha} p}{\int_{b_{1}}^{b_{2}}|\hbar(p)| \diamond_{\alpha} p}\right) \leq \frac{\int_{b_{1}}^{b_{2}}|\hbar(p)| \chi(\zeta(p)) \diamond_{\alpha} p}{\int_{b_{1}}^{b_{2}}|\hbar(p)| \diamond_{\alpha} p} .
$$

Da Cruz et al. in [11] described diamond integral (a generalization of diamond- $\alpha$ integral) in terms of "approximate symmetric integral" on time scales $\mathbb{T}$. Bibi et al. proved Jensen's inequality related to diamond integrals in [5].

Theorem 4 Suppose $b_{1}, b_{2} \in \mathbb{T}$ with $b_{1}<b_{2}$, and $\hbar \in C\left(\left[b_{1}, b_{2}\right]_{\mathbb{T}}, \mathbb{R}\right)$ which satisfies $\int_{b_{1}}^{b_{2}} \hbar(p) \diamond p \neq 0$. Also assume $\zeta \in C\left(\left[b_{1}, b_{2}\right]_{\mathbb{T}}, E\right)$ and a convex function $\chi \in C(E, \mathbb{R})$, where $E=[w, W]$ with $w=\min _{p \in \chi:\left[b_{1}, b_{2}\right]_{\mathbb{T}}} \zeta(p), W=\max _{p \in \chi:\left[b_{1}, b_{2}\right]_{\mathbb{T}}} \zeta(p)$, then

$$
\chi\left(\frac{\int_{b_{1}}^{b_{2}}|\hbar(p)| \zeta(p) \diamond p}{\int_{b_{1}}^{b_{2}}|\hbar(p)| \diamond p}\right) \leq \frac{\int_{b_{1}}^{b_{2}}|\hbar(p)| \chi(\zeta(p)) \diamond p}{\int_{b_{1}}^{b_{2}}|\hbar(p)| \diamond p} .
$$


Under the suppositions of Theorem 4, inequality (16) produces the linear functional

$$
J(\chi)=\frac{\int_{b_{1}}^{b_{2}}|\hbar(p)| \chi(\zeta(p)) \diamond p}{\int_{b_{1}}^{b_{2}} \hbar(p) \diamond p}-\chi\left(\frac{\int_{b_{1}}^{b_{2}} \hbar(p) \zeta(p) \diamond p}{\int_{b_{1}}^{b_{2}} \hbar(p) \diamond p}\right) .
$$

Remark 4 By Theorem 4, we conclude that $J(\chi)=0$ if $\chi$ is a constant function or an identity function and $J(\chi) \geq 0$ for the group of convex functions.

In the same manner, the linear functionals for (14) and (15) can be obtained.

\section{Generalized Jensen-type functional by extended Montgomery identity via Taylor's formula I}

Theorem 5 Let $n \in N$ such that $n \geq 2$ and all the suppositions of Theorem 4 be satisfied. If $\chi$ is a convex function defined on $\left[b_{1}, b_{2}\right]$ such that $\chi^{(n-1)}$ is absolutely continuous, then $\forall t \in\left[b_{1}, b_{2}\right]$ we have

$$
\begin{aligned}
J(\chi)= & \frac{1}{b_{2}-b_{1}} \sum_{\mathfrak{z}=0}^{n-2}\left(\frac{1}{\mathfrak{z} !(\mathfrak{z}+2)}\right)\left(\chi^{(\mathfrak{z}+1)}\left(b_{1}\right) J\left(t-b_{1}\right)^{\mathfrak{z}+2}-\chi^{(\mathfrak{z}+1)}\left(b_{2}\right) J\left(t-b_{2}\right)^{\mathfrak{z}+2}\right) \\
& +\frac{1}{(n-1) !} \int_{b_{1}}^{b_{2}} J\left(R_{n}(t, s)\right) \chi^{n}(s) d s,
\end{aligned}
$$

where $R_{n}(t, s)$ is defined in (6) and

$$
J\left(R_{n}(t, s)\right)=\frac{\int_{b_{1}}^{b_{2}}|\hbar(\tau)| R_{n}(\chi(\tau), s) \diamond \tau}{\int_{b_{1}}^{b_{2}}|\hbar(\tau)| \diamond \tau}-R_{n}\left(\left(\frac{\int_{b_{1}}^{b_{2}}|\hbar(\tau)| \chi(\tau) \diamond \tau}{\int_{b_{1}}^{b_{2}}|\hbar(\tau)| \diamond \tau}\right), s\right) .
$$

Proof Putting (5) in (17), we obtain

$$
\begin{aligned}
J(\chi)= & J\left(\frac{1}{b_{2}-b_{1}} \int_{b_{1}}^{b_{2}} \chi(p) d p+\sum_{\mathfrak{z}=0}^{n-2} \frac{\chi^{(\mathfrak{z}+1)}\left(b_{1}\right)}{\mathfrak{z} !(\mathfrak{z}+2)} \frac{\left(t-b_{1}\right)^{\mathfrak{z}+2}}{b_{2}-b_{1}}\right. \\
& \left.-\sum_{\mathfrak{z}=0}^{n-2} \frac{\chi^{(\mathfrak{z}+1)}\left(b_{2}\right)}{\mathfrak{z} !(\mathfrak{z}+2)} \frac{\left(t-b_{2}\right)^{\mathfrak{z}+2}}{b_{2}-b_{1}}+\frac{1}{(n-1) !} \int_{b_{1}}^{b_{2}} R_{n}(t, s) \chi^{(n)}(s) d s\right) .
\end{aligned}
$$

The linearity of the functional $J(\cdot)$ gives us

$$
\begin{aligned}
J(\chi)= & J\left(\frac{1}{b_{2}-b_{1}} \int_{b_{1}}^{b_{2}} \chi(p) d p\right)+J\left(\sum_{\mathfrak{z}=0}^{n-2} \frac{\chi^{(\mathfrak{z}+1)}\left(b_{1}\right)}{\mathfrak{z} !(\mathfrak{z}+2)} \frac{\left(t-b_{1}\right)^{\mathfrak{z}+2}}{b_{2}-b_{1}}\right) \\
& -J\left(\sum_{\mathfrak{z}=0}^{n-2} \frac{\chi^{(\mathfrak{z}+1)}\left(b_{2}\right)}{\mathfrak{z} !(\mathfrak{z}+2)} \frac{\left(t-b_{2}\right)^{\mathfrak{z}+2}}{b_{2}-b_{1}}\right)+J\left(\frac{1}{(n-1) !} \int_{b_{1}}^{b_{2}} R_{n}(t, s) \chi^{(n)}(s) d s\right) \\
= & \sum_{\mathfrak{z}=0}^{n-2} \frac{1}{\mathfrak{z} !(\mathfrak{z}+2)\left(b_{2}-b_{1}\right)}\left(\chi^{(\mathfrak{z}+1)}\left(b_{1}\right) J\left(t-b_{1}\right)^{\mathfrak{z}+2}-\chi^{(\mathfrak{z}+1)}\left(b_{2}\right) J\left(t-b_{2}\right)^{\mathfrak{z}+2}\right) \\
& +\frac{1}{(n-1) !} \int_{b_{1}}^{b_{2}} J\left(R_{n}(t, s)\right) \chi^{(n)}(s) d s .
\end{aligned}
$$


Theorem 6 Let all the suppositions of Theorem 5 be satisfied and

$$
J\left(R_{n}(t, s)\right) \geq 0
$$

for all $s \in\left[b_{1}, b_{2}\right]$. If $\chi$ is $n$-convex such that $\chi^{(n-1)}$ is absolutely continuous, then we have that

$$
J(\chi) \geq J(U)
$$

holds, where

$$
U(t)=\frac{1}{b_{2}-b_{1}} \sum_{\mathfrak{z}=0}^{n-2}\left(\frac{1}{\mathfrak{z} !(\mathfrak{z}+2)}\right)\left(\chi^{(\mathfrak{z}+1)}\left(b_{1}\right)\left(t-b_{1}\right)^{\mathfrak{z}+2}-\chi^{(\mathfrak{z}+1)}\left(b_{2}\right)\left(t-b_{2}\right)^{\mathfrak{z}+2}\right) .
$$

The reverse inequality in (21) gives rise to the reverse inequality in (22).

Proof Since $\chi^{(n-1)}$ is absolutely continuous on $\left[b_{1}, b_{2}\right]$, therefore $\chi^{n}$ exists almost everywhere. Now the $n$-convexity of $\chi$ implies that $\chi^{n}(t) \geq 0, \forall t \in\left[b_{1}, b_{2}\right]$; this fact together with (21) implies that

$$
J\left(R_{n}(t, s)\right) \chi^{n}(s) \geq 0
$$

for all $s \in\left[b_{1}, b_{2}\right]$. Thus

$$
\frac{1}{(n-1) !} \int_{b_{1}}^{b_{2}} J\left(R_{n}(t, s)\right) \chi^{n}(s) d s \geq 0 .
$$

Using (25) in (18), we get

$$
J(\chi)-\frac{1}{b_{2}-b_{1}} \sum_{\mathfrak{z}=0}^{n-2}\left(\frac{1}{\mathfrak{z} !(\mathfrak{z}+2)}\right)\left(\chi^{(\mathfrak{z}+1)}\left(b_{1}\right) J\left(t-b_{1}\right)^{\mathfrak{z}+2}-\chi^{(\mathfrak{z}+1)}\left(b_{2}\right) J\left(t-b_{2}\right)^{\mathfrak{z}+2}\right) \geq 0 .
$$

The linearity of $J(\cdot)$ yields

$$
\begin{aligned}
& J(\chi)-J\left(\frac{1}{b_{2}-b_{1}} \sum_{\mathfrak{z}=0}^{n-2}\left(\frac{1}{\mathfrak{z} !(\mathfrak{z}+2)}\right)\left(\chi^{(\mathfrak{z}+1)}\left(b_{1}\right)\left(t-b_{1}\right)^{\mathfrak{z}+2}-\chi^{(\mathfrak{z}+1)}\left(b_{2}\right)\left(t-b_{2}\right)^{\mathfrak{z}+2}\right)\right) \\
& \quad \geq 0,
\end{aligned}
$$

which is the required result. The reverse inequality in (21) gives rise to the reverse inequality in (24); therefore, the reverse inequality in (22) is obtained.

Theorem 7 Let all the suppositions of Theorem 6 be valid, and let $U$, defined in (23), be convex on $\left[b_{1}, b_{2}\right]$. Then we have

$$
J(\chi(t)) \geq 0
$$

for all $t \in\left[b_{1}, b_{2}\right]$. 
Proof Since $U(t)$ is convex $\forall t \in\left[b_{1}, b_{2}\right]$, thus by means of Remark 4 , we get $J(U(t)) \geq 0$. As a result, (22) implies $J(\chi(t)) \geq 0$.

Remark 5 The generalized form of Theorem 4 is Theorem 7.

\section{Generalized Jensen-type functional by extended Montgomery identity via Taylor's formula II}

Theorem 8 Let $n \in N$ such that $n \geq 2$ and all the suppositions of Theorem 4 be satisfied. If $\chi$ is a convex function defined on $\left[b_{1}, b_{2}\right]$ such that $\chi^{(n-1)}$ is absolutely continuous, then $\forall t \in\left[b_{1}, b_{2}\right]$ we have $\forall t \in\left[b_{1}, b_{2}\right]$

$$
\begin{aligned}
J(\chi)= & \frac{1}{b_{2}-b_{1}} \sum_{\mathfrak{z}=0}^{n-2}\left(\frac{1}{(\mathfrak{z}+2) !}\right)\left(J\left(\chi^{(\mathfrak{z}+1)}(t)\left(b_{1}-t\right)^{\mathfrak{z}+2}\right)-J\left(\chi^{(\mathfrak{z}+1)}(t)\left(b_{2}-t\right)^{\mathfrak{z}+2}\right)\right) \\
& +\frac{1}{(n-1) !} \int_{p}^{q} J\left(\hat{R}_{n}(t, s)\right) \chi^{n}(s) d s,
\end{aligned}
$$

where $\hat{R}_{n}$ is defined in (8) and

$$
J\left(\hat{R}_{n}(t, s)\right)=\frac{\int_{b_{1}}^{b_{2}}|\hbar(\tau)| \hat{R}_{n}(\chi(\tau), s) \diamond \tau}{\int_{b_{1}}^{b_{2}}|\hbar(\tau)| \diamond \tau}-\hat{R}_{n}\left(\left(\frac{\int_{b_{1}}^{b_{2}}|\hbar(\tau)| \chi(\tau) \diamond \tau}{\int_{b_{1}}^{b_{2}}|\hbar(\tau)| \diamond \tau}\right), s\right) .
$$

Proof Putting (7) in (17), we obtain

$$
\begin{aligned}
J(\chi)= & J\left(\frac{1}{b_{2}-b_{1}} \int_{b_{1}}^{b_{2}} \chi(p) d p+\sum_{\mathfrak{z}=0}^{n-2} \chi^{(\mathfrak{z}+1)}(t) \frac{\left(b_{1}-t\right)^{\mathfrak{z}+2}-\left(b_{2}-t\right)^{\mathfrak{z}+2}}{(\mathfrak{z}+2) !\left(b_{2}-b_{1}\right)}\right. \\
& \left.+\frac{1}{(n-1) !} \int_{b_{1}}^{b_{2}} \hat{R}_{n}(t, s) \chi^{(n)}(s) d s\right) .
\end{aligned}
$$

By using the linearity of the functional $J(\cdot)$, we get

$$
\begin{aligned}
J(\chi)= & J\left(\frac{1}{b_{2}-b_{1}} \int_{b_{1}}^{b_{2}} \chi(p) d p\right)+J\left(\sum_{\mathfrak{z}=0}^{n-2} \chi^{(\mathfrak{z}+1)}(t) \frac{\left(b_{1}-t\right)^{\mathfrak{z}+2}-\left(b_{2}-t\right)^{\mathfrak{z}+2}}{(\mathfrak{z}+2) !\left(b_{2}-b_{1}\right)}\right) \\
& +J\left(\frac{1}{(n-1) !} \int_{b_{1}}^{b_{2}} \hat{R}_{n}(t, s) \chi^{(n)}(s) d s\right) \\
= & \sum_{\mathfrak{z}=0}^{n-2} \frac{1}{(\mathfrak{z}+2) !\left(b_{2}-b_{1}\right)} \times J\left(\left(\chi^{(\mathfrak{z}+1)}(t)\left(b_{1}-t\right)^{\mathfrak{z}+2}\right)-\left(\chi^{(\mathfrak{z}+1)}(t)\left(b_{2}-t\right)^{\mathfrak{z}+2}\right)\right) \\
& +\frac{1}{(n-1) !} \int_{b_{1}}^{b_{2}} J\left(\hat{R}_{n}(t, s)\right) \chi^{(n)}(s) d s .
\end{aligned}
$$

Theorem 9 Let all the suppositions of Theorem 8 be satisfied and

$$
J\left(\hat{R}_{n}(t, s)\right) \geq 0
$$


for all $s \in\left[b_{1}, b_{2}\right]$. If $\chi$ is $n$-convex such that $\chi^{(n-1)}$ is absolutely continuous, then we have

$$
J(\chi) \geq J(U)
$$

holds, where

$$
U(t)=\frac{1}{b_{2}-b_{1}} \sum_{\mathfrak{z}=0}^{n-2}\left(\frac{1}{(\mathfrak{z}+2) !}\right)\left(\left(\chi^{(\mathfrak{z}+1)}(t)\left(b_{1}-t\right)^{\mathfrak{z}+2}\right)-\left(\chi^{(\mathfrak{z}+1)}(t)\left(b_{2}-t\right)^{\mathfrak{z}+2}\right)\right) .
$$

Proof Since $\chi^{(n-1)}$ is absolutely continuous on $\left[b_{1}, b_{2}\right]$, therefore $\chi^{n}$ exists almost everywhere. Now the $n$-convexity of $\chi$ implies that $\chi^{n}(t) \geq 0, \forall t \in\left[b_{1}, b_{2}\right]$; this fact together with (21) implies that

$$
J\left(\hat{R}_{n}(t, s)\right) \chi^{n}(s) \geq 0
$$

for all $s \in\left[b_{1}, b_{2}\right]$. Thus

$$
\frac{1}{(n-1) !} \int_{b_{1}}^{b_{2}} J\left(\hat{R}_{n}(t, s)\right) \chi^{n}(s) d s \geq 0
$$

Using (36) in (29), we get

$$
J(\chi)-\frac{1}{b_{2}-b_{1}} \sum_{\mathfrak{z}=0}^{n-2}\left(\frac{1}{(\mathfrak{z}+2) !}\right)\left(J\left(\chi^{(\mathfrak{z}+1)}(t)\left(b_{1}-t\right)^{\mathfrak{z}+2}\right)-J\left(\chi^{(\mathfrak{z}+1)}(t)\left(b_{2}-t\right)^{\mathfrak{z}+2}\right)\right) \geq 0 .
$$

By the linearity of $J(\cdot)$, we get

$$
J(\chi)-J\left(\frac{1}{b_{2}-b_{1}} \sum_{\mathfrak{z}=0}^{n-2}\left(\frac{1}{(\mathfrak{z}+2) !}\right)\left(\chi^{(\mathfrak{z}+1)}(t)\left(b_{1}-t\right)^{\mathfrak{z}+2}-\chi^{(\mathfrak{z}+1)}(t)\left(b_{2}-t\right)^{\mathfrak{z}+2}\right)\right) \geq 0,
$$

which is the required result.

The reverse inequality in (32) gives rise to the reverse inequality in (35); therefore, the reverse inequality in (33) is obtained.

Theorem 10 Let all the supposition of Theorem 9 be valid, and let $U$, defined in (34), be convex on $\left[b_{1}, b_{2}\right]$. Then we have

$$
J(\chi(t)) \geq 0
$$

for all $t \in\left[b_{1}, b_{2}\right]$.

Proof Since $U(t)$ is convex $\forall t \in\left[b_{1}, b_{2}\right]$, thus by means of Remark 4 , we get $J(U(t)) \geq 0$. As a result, (33) implies $J(\chi(t)) \geq 0$.

Remark 6 The generalized form of Theorem 4 is Theorem 10. 


\section{Bounds for identities associated with generalization of Jensen-type functional}

Assume $\mathfrak{f}, v:\left[b_{1}, b_{2}\right] \rightarrow \mathbb{R}$ as Lebesgue integrable functions, then the Čebyšev functional is

$$
\Omega(\mathfrak{f}, v)=\frac{1}{b_{2}-b_{1}} \int_{b_{1}}^{b_{2}} \mathfrak{f}(t) v(t) d t-\frac{1}{b_{2}-b_{1}} \int_{b_{1}}^{b_{2}} \mathfrak{f}(t) d t \frac{1}{b_{2}-b_{1}} \int_{b_{1}}^{b_{2}} v(t) d t .
$$

The following theorems were proved by Cerone and Dragomir in [10].

Theorem 11 Consider a Lebesgue integrable function $\mathfrak{f}:\left[b_{1}, b_{2}\right] \rightarrow \mathbb{R}$ and an absolutely continuous function $v:\left[b_{1}, b_{2}\right] \rightarrow \mathbb{R}$ with $\left(\cdot-b_{1}\right)\left(b_{2}-\cdot\right)\left[v^{\prime}\right]^{2} \in L\left[b_{1}, b_{2}\right]$. Then

$$
|\Omega(\mathfrak{f}, v)| \leq \frac{1}{\sqrt{2}}[\Omega(\mathfrak{f}, \mathfrak{f})]^{\frac{1}{2}} \frac{1}{\sqrt{b_{2}-b_{1}}}\left(\int_{b_{1}}^{b_{2}}\left(y-b_{1}\right)\left(b_{2}-y\right)\left[v^{\prime}(y)\right]^{2} d y\right)^{\frac{1}{2}}
$$

The constant $\frac{1}{\sqrt{2}}$ in (41) is the best possible.

Theorem 12 If $v:\left[b_{1}, b_{2}\right] \rightarrow \mathbb{R}$ is monotonic nondecreasing on $\left[b_{1}, b_{2}\right]$ and $\mathfrak{f}:\left[b_{1}, b_{2}\right] \rightarrow \mathbb{R}$ is absolutely continuous with $\mathfrak{f}^{\prime} \in L_{\infty}\left[b_{1}, b_{2}\right]$. Then we have

$$
|\Omega(\mathfrak{f}, v)| \leq \frac{1}{2\left(b_{2}-b_{1}\right)}\left\|\mathfrak{f}^{\prime}\right\|_{\infty} \int_{b_{1}}^{b_{2}}\left(y-b_{1}\right)\left(b_{2}-y\right) d h(y) .
$$

The constant $\frac{1}{2}$ in (42) is the best possible.

Let us denote

$$
\varrho(s)=J\left(R_{n}(t, s)\right), \quad s \in\left[b_{1}, b_{2}\right]
$$

and

$$
\hat{\varrho}(s)=J\left(\hat{R}_{n}(t, s)\right), \quad s \in\left[b_{1}, b_{2}\right]
$$

Now, the Čebyšev functionals defined as

$$
\begin{aligned}
& \Omega(\varrho, \varrho)=\frac{1}{b_{2}-b_{1}} \int_{b_{1}}^{b_{2}} \varrho^{2}(s) d s-\left(\frac{1}{b_{2}-b_{1}} \int_{b_{1}}^{b_{2}} \varrho(s) d s\right)^{2} . \\
& \Omega(\hat{\varrho}, \hat{\varrho})=\frac{1}{b_{2}-b_{1}} \int_{b_{1}}^{b_{2}} \hat{\varrho}^{2}(s) d s-\left(\frac{1}{b_{2}-b_{1}} \int_{b_{1}}^{b_{2}} \hat{\varrho}(s) d s\right)^{2} .
\end{aligned}
$$

Theorem 13 Let $n \in N$ such that $n \geq 2$ and all the suppositions of Theorem 4 be satisfied. If $\chi$ is a convex function defined on $\left[b_{1}, b_{2}\right]$ such that $\chi^{(n-1)}$ is absolutely continuous, (. $\left.b_{1}\right)\left(b_{2}-\cdot\right)\left[\chi^{(n+1)}\right]^{2} \in L\left[b_{1}, b_{2}\right]$, and $R_{n}, \hat{R}_{n}, \varrho, \hat{\varrho}$, and $\Omega$ be defined in (6), (8), (43), (44), (45), 
and (46), respectively, then $\forall t \in\left[b_{1}, b_{2}\right]$ we have

$$
\begin{aligned}
J(\chi)= & \frac{1}{b_{2}-b_{1}} \sum_{\mathfrak{z}=0}^{n-2}\left(\frac{1}{\mathfrak{z} !(\mathfrak{z}+2)}\right)\left(\chi^{(\mathfrak{z}+1)}\left(b_{1}\right) J\left(t-b_{1}\right)^{\mathfrak{z}+2}-\chi^{(\mathfrak{z}+1)}\left(b_{2}\right) J\left(t-b_{2}\right)^{\mathfrak{z}+2}\right) \\
& +\frac{\chi^{(\mathfrak{z}-1)}\left(b_{2}\right)-\chi^{(\mathfrak{z}-1)}\left(b_{1}\right)}{\left(b_{2}-b_{1}\right)(n-1) !} \int_{b_{1}}^{b_{2}} \varrho(s) d s+H_{n}\left(\chi ; b_{1}, b_{2}\right)
\end{aligned}
$$

and

$$
\begin{aligned}
J(\chi)= & \frac{1}{b_{2}-b_{1}} \sum_{\mathfrak{z}=0}^{n-2}\left(\frac{1}{(\mathfrak{z}+2) !}\right)\left(J\left(\chi^{(\mathfrak{z}+1)}(t)\left(b_{1}-t\right)^{\mathfrak{z}+2}\right)-J\left(\chi^{(\mathfrak{z}+1)}(t)\left(b_{2}-t\right)^{\mathfrak{z}+2}\right)\right) \\
& +\frac{\chi^{(\mathfrak{z}-1)}\left(b_{2}\right)-\chi^{(\mathfrak{z}-1)}\left(b_{1}\right)}{\left(b_{2}-b_{1}\right)(n-1) !} \int_{b_{1}}^{b_{2}} \hat{\varrho}(s) d s+\hat{H}_{n}\left(\chi ; b_{1}, b_{2}\right) .
\end{aligned}
$$

The remainders $H_{n}\left(\chi ; b_{1}, b_{2}\right)$ and $\hat{H}_{n}\left(\chi ; b_{1}, b_{2}\right)$ satisfy the approximation

$$
\begin{aligned}
& \left|H_{n}\left(\chi ; b_{1}, b_{2}\right)\right| \leq \frac{[\Omega(\varrho, \varrho)]^{\frac{1}{2}}}{(n-1) !} \sqrt{\frac{b_{2}-b_{1}}{2}}\left|\int_{b_{1}}^{b_{2}}\left(s-b_{1}\right)\left(b_{2}-s\right)\left[\chi^{(n+1)}(s)\right]^{2} d s\right|^{\frac{1}{2}}, \\
& \left|\hat{H}_{n}\left(\chi ; b_{1}, b_{2}\right)\right| \leq \frac{[\Omega(\hat{\varrho}, \hat{\varrho})]^{\frac{1}{2}}}{(n-1) !} \sqrt{\frac{b_{2}-b_{1}}{2}}\left|\int_{b_{1}}^{b_{2}}\left(s-b_{1}\right)\left(b_{2}-s\right)\left[\chi^{(n+1)}(s)\right]^{2} d s\right|^{\frac{1}{2}} .
\end{aligned}
$$

Proof Applying Theorem 11 for $\mathfrak{f} \rightarrow \varrho$ and $v \rightarrow \chi^{(n)}$, we obtain

$$
\begin{gathered}
\left|\frac{1}{b_{2}-b_{1}} \int_{b_{1}}^{b_{2}} \varrho(s) \chi^{(n)}(s) d s-\frac{1}{b_{2}-b_{1}} \int_{b_{1}}^{b_{2}} \varrho(s) d s \frac{1}{b_{2}-b_{1}} \int_{b_{1}}^{b_{2}} \chi^{(n)}(s) d s\right| \\
\leq \frac{1}{\sqrt{2}}[\Omega(\varrho, \varrho)]^{\frac{1}{2}} \frac{1}{\sqrt{b_{2}-b_{1}}}\left|\int_{b_{1}}^{b_{2}}\left(s-b_{1}\right)\left(b_{2}-s\right)\left[\chi^{(n+1)}(s)\right]^{2} d s\right|^{\frac{1}{2}} .
\end{gathered}
$$

Now, dividing both sides of $(51)$ by $(n-1)$ ! and multiplying by $\left(b_{2}-b_{1}\right)$, we get

$$
\begin{aligned}
& \left|\frac{1}{(n-1) !} \int_{b_{1}}^{b_{2}} \varrho(s) \chi^{(n)}(s) d s-\frac{1}{(n-1) !} \int_{b_{1}}^{b_{2}} \varrho(s) d s \frac{\chi^{(n-1)}\left(b_{2}\right)-\chi^{(n-1)}\left(b_{1}\right)}{\left(b_{2}-b_{1}\right)}\right| \\
& \quad \leq \sqrt{\frac{b_{2}-b_{1}}{2}} \frac{[\Omega(\varrho, \varrho)]^{\frac{1}{2}}}{(n-1) !}\left|\int_{b_{1}}^{b_{2}}\left(s-b_{1}\right)\left(b_{2}-s\right)\left[\chi^{(n+1)}(s)\right]^{2} d s\right|^{\frac{1}{2}} .
\end{aligned}
$$

By denoting

$$
\begin{aligned}
H_{n}\left(\chi ; b_{1}, b_{2}\right)= & \frac{1}{(n-1) !} \int_{b_{1}}^{b_{2}} \varrho(s) \chi^{(n)}(s) d s \\
& -\frac{1}{(n-1) !} \int_{b_{1}}^{b_{2}} \varrho(s) d s \frac{\chi^{(n-1)}\left(b_{2}\right)-\chi^{(n-1)}\left(b_{1}\right)}{\left(b_{2}-b_{1}\right)}
\end{aligned}
$$

in (52), we have (49). Hence, we obtain

$$
\frac{1}{(n-1) !} \int_{b_{1}}^{b_{2}} \varrho(s) \chi^{(n)}(s) d s=\frac{\chi^{(n-1)}\left(b_{2}\right)-\chi^{(n-1)}\left(b_{1}\right)}{\left(b_{2}-b_{1}\right)(n-1) !} \int_{b_{1}}^{b_{2}} \varrho(s) d s+H_{n}\left(\chi ; b_{1}, b_{2}\right)
$$


where the remainder $H_{n}\left(\chi ; b_{1}, b_{2}\right)$ satisfies approximation (49). Now from (18) we obtain (47).

Similarly, from identity (29), we get (50).

Using Theorem 12, the Grüss-type inequality is obtained in the following theorem.

Theorem 14 Let $n \in N$ such that $n \geq 2$ and all the suppositions of Theorem 4 be satisfied. If $\chi$ is a convex function defined on $\left[b_{1}, b_{2}\right]$ such that $\chi^{(n)}$ is absolutely continuous, $\chi^{(n+1)} \geq 0$ on $\left[b_{1}, b_{2}\right]$, and the functions $\varrho$, $\hat{\varrho}$, and $\Omega$ are defined by (43), (44), (45), and (46), respectively. Then we have (47), and the remainder $H_{n}\left(\chi ; b_{1}, b_{2}\right)$ satisfies the bound

$$
\begin{aligned}
\left|H_{n}\left(\chi ; b_{1}, b_{2}\right)\right| \leq & \frac{\left(b_{2}-b_{1}\right)}{(n-1) !}\left\|\varrho^{\prime}\right\|_{\infty} \\
& \times\left\{\frac{\chi^{(n-1)}\left(b_{2}\right)+\chi^{(n-1)}\left(b_{1}\right)}{2}-\frac{\chi^{(n-2)}\left(b_{2}\right)-\chi^{(n-2)}\left(b_{1}\right)}{b_{2}-b_{1}}\right\},
\end{aligned}
$$

where as representation (48) and the remainder $\hat{H}_{n}\left(\chi ; b_{1}, b_{2}\right)$ satisfies the approximation

$$
\begin{aligned}
\left|\hat{H}_{n}\left(\chi ; b_{1}, b_{2}\right)\right| \leq & \frac{\left(b_{2}-b_{1}\right)}{(n-1) !}\left\|\varrho^{\prime}\right\|_{\infty} \\
& \times\left\{\frac{\chi^{(n-1)}\left(b_{2}\right)+\chi^{(n-1)}\left(b_{1}\right)}{2}-\frac{\chi^{(n-2)}\left(b_{2}\right)-\chi^{(n-2)}\left(b_{1}\right)}{b_{2}-b_{1}}\right\} .
\end{aligned}
$$

Proof Using Theorem 12 for $f \rightarrow \varrho$ and $v \rightarrow \chi^{(n)}$, we get

$$
\begin{aligned}
& \left|\frac{1}{b_{2}-b_{1}} \int_{b_{1}}^{b_{2}} \varrho(s) \chi^{(n)}(s) d s-\frac{1}{b_{2}-b_{1}} \int_{b_{1}}^{b_{2}} \varrho(s) d s \frac{1}{b_{2}-b_{1}} \int_{b_{1}}^{b_{2}} \chi^{(n)}(s) d s\right| \\
& \quad \leq \frac{1}{2\left(b_{2}-b_{1}\right)}\left\|\varrho^{\prime}\right\|_{\infty} \int_{b_{1}}^{b_{2}}\left(s-b_{1}\right)\left(b_{2}-s\right) \chi^{(n+1)}(s) d s .
\end{aligned}
$$

Since

$$
\begin{aligned}
& \int_{b_{1}}^{b_{2}}\left(s-b_{1}\right)\left(b_{2}-s\right) \chi^{(n+1)}(s) d s \\
& \quad=\int_{b_{1}}^{b_{2}}\left[2 s-\left(b_{1}+b_{2}\right)\right] \chi^{(n)}(s) d s \\
& \quad=\left(b_{2}-b_{1}\right)\left[\chi^{(n-1)}\left(b_{2}\right)+\chi^{(n-1)}\left(b_{1}\right)\right]-2\left(\chi^{(n-2)}\left(b_{2}\right)-\chi^{(n-2)}\left(b_{1}\right)\right),
\end{aligned}
$$

dividing both sides of (57) by $(n-1)$ ! and multiplying by $\left(b_{2}-b_{1}\right)$, we have

$$
\begin{aligned}
& \left|\frac{1}{(n-1) !} \int_{b_{1}}^{b_{2}} \varrho(s) \chi^{(n)}(s) d s-\int_{b_{1}}^{b_{2}} \varrho(s) d s \frac{\chi^{(n-1)}\left(b_{2}\right)-\chi^{(n-1)}\left(b_{1}\right)}{\left(b_{2}-b_{1}\right)(n-1) !}\right| \\
& \quad \leq \frac{\left(b_{2}-b_{1}\right)}{(n-1) !}\left\|\varrho^{\prime}\right\|_{\infty}\left[\frac{\left[\chi^{(n-1)}\left(b_{2}\right)+\chi^{(n-1)}\left(b_{1}\right)\right]}{2}-\frac{\left(\chi^{(n-2)}\left(b_{2}\right)-\chi^{(n-2)}\left(b_{1}\right)\right)}{b_{2}-b_{1}}\right] .
\end{aligned}
$$


Using (18), we get

$$
\begin{aligned}
\mid J(\chi) & -\frac{1}{b_{2}-b_{1}} \sum_{\mathfrak{z}=0}^{n-2}\left(\frac{1}{\mathfrak{z} !(\mathfrak{z}+2)}\right)\left(\chi^{(\mathfrak{z}+1)}\left(b_{1}\right) J\left(t-b_{1}\right)^{\mathfrak{z}+2}-\chi^{(\mathfrak{z}+1)}\left(b_{2}\right) J\left(t-b_{2}\right)^{\mathfrak{z}+2}\right) \\
& -\frac{1}{(n-1) !} \int_{b_{1}}^{b_{2}} \varrho(s) d s \frac{\chi^{(n-1)}\left(b_{2}\right)-\chi^{(n-1)}\left(b_{1}\right)}{b_{2}-b_{1}} \mid \\
& \leq \frac{\left(b_{2}-b_{1}\right)}{(n-1) !}\left\|\varrho^{\prime}\right\|_{\infty}\left[\frac{\left[\chi^{(n-1)}\left(b_{2}\right)+\chi^{(n-1)}\left(b_{1}\right)\right]}{2}-\frac{\left(\chi^{(n-2)}\left(b_{2}\right)-\chi^{(n-2)}\left(b_{1}\right)\right)}{b_{2}-b_{1}}\right] .
\end{aligned}
$$

Now, using (47), we deduce (55)

$$
\begin{aligned}
\left|\hat{H}_{n}\left(\chi ; b_{1}, b_{2}\right)\right| \leq & \frac{\left(b_{2}-b_{1}\right)}{(n-1) !}\left\|\varrho^{\prime}\right\|_{\infty} \\
& \times\left[\frac{\left[\chi^{(n-1)}\left(b_{2}\right)+\chi^{(n-1)}\left(b_{1}\right)\right]}{2}-\frac{\left[\chi^{(n-2)}\left(b_{2}\right)-\chi^{(n-2)}\left(b_{1}\right)\right]}{b_{2}-b_{1}}\right] .
\end{aligned}
$$

Similarly, using (29) instead of (18), we get (56).

The Ostrowski-type inequality allied to generalized Jensen's inequality is specified by the following theorem.

Theorem 15 Assume that the conditions of Theorems 5 and 8 are satisfied. Consider a specific pair $\left(r_{1}, r_{2}\right)$ of conjugate exponents, i.e., $1 \leq r_{1}, r_{2} \leq \infty, \frac{1}{r_{1}}+\frac{1}{r_{2}}=1$. For $n \in N$, assume that the function $\left|\chi^{(n)}\right|^{r_{1}}:\left[b_{1}, b_{2}\right] \rightarrow \mathbb{R}$ is Riemann integrable for $n \geq 2$. Then

(i)

$$
\begin{aligned}
& \left|J(\chi)-\frac{1}{b_{2}-b_{1}} \sum_{\mathfrak{z}=0}^{n-2}\left(\frac{1}{\mathfrak{z} !(\mathfrak{z}+2)}\right)\left(\chi^{(\mathfrak{z}+1)}\left(b_{1}\right) J\left(t-b_{1}\right)^{\mathfrak{z}+2}-\chi^{(\mathfrak{z}+1)}\left(b_{2}\right) J\left(t-b_{2}\right)^{\mathfrak{z}+2}\right)\right| \\
& \quad \leq \frac{1}{(n-1) !}\left\|\chi^{(n)}\right\|_{r_{1}}\left(\int_{b_{1}}^{b_{2}}\left|J\left(R_{n}(t, s)\right)\right|^{r_{2}} d s\right)^{\frac{1}{r_{2}}}
\end{aligned}
$$

The constant on the right-hand side of (61) is sharp for $1<r_{1} \leq \infty$ and best possible for $r_{1}=1$.

$$
\begin{gathered}
\text { (ii) }\left|J(\chi)-\frac{1}{b_{2}-b_{1}} \sum_{\mathfrak{z}=0}^{n-2}\left(\frac{1}{\mathfrak{z} !(\mathfrak{z}+2)}\right)\left(J\left(\chi^{(\mathfrak{z}+1)}(t)\left(b_{1}-t\right)^{\mathfrak{z}+2}\right)-J\left(\chi^{(\mathfrak{z}+1)}(t)\left(b_{2}-t\right)^{\mathfrak{z}+2}\right)\right)\right| \\
\leq \frac{1}{(n-1) !}\left\|\chi^{(n)}\right\|_{r_{1}}\left(\int_{b_{1}}^{b_{2}}\left|J\left(\hat{R}_{n}(t, s)\right)\right|^{r_{2}} d s\right)^{\frac{1}{r_{2}}} .
\end{gathered}
$$

The constant on the right-hand side of (62) is sharp for $1<r_{1} \leq \infty$ and best possible for $r_{1}=1$.

Proof (i) Let us take

$$
\varrho(s)=\frac{1}{(n-1) !} J\left(R_{n}(t, s)\right)
$$


Using (18) and Hölder's inequality, we get

$$
\begin{aligned}
& \left|J(\chi)-\frac{1}{b_{2}-b_{1}} \sum_{\mathfrak{z}=0}^{n-2}\left(\frac{1}{\mathfrak{z} !(\mathfrak{z}+2)}\right)\left(\chi^{(\mathfrak{z}+1)}\left(b_{1}\right) J\left(t-b_{1}\right)^{\mathfrak{z}+2}-\chi^{(\mathfrak{z}+1)}\left(b_{2}\right) J\left(t-b_{2}\right)^{\mathfrak{z}+2}\right)\right| \\
& \quad=\left|\int_{b_{1}}^{b_{2}} \varrho(s) \chi^{n}(s) d s\right| \leq\left\|\chi^{(n)}\right\|_{r_{1}}\left(\int_{b_{1}}^{b_{2}}|\varrho(s)|^{r_{2}} d s\right)^{\frac{1}{r_{2}}} .
\end{aligned}
$$

The sharpness of the constant $\left(\int_{b_{1}}^{b_{2}}|\varrho(s)|^{r_{2}} d s\right)^{\frac{1}{r_{2}}}$ is proved by considering a function $\chi$ for which the inequality in (61) is obtained.

For $1<r_{1}<\infty$, we take

$$
\chi^{(n)}(s)=\operatorname{sgn} \varrho(s)|\varrho(s)|^{\frac{1}{r_{1}-1}}
$$

For $r_{1}=\infty$, take $\chi^{(n)}(s)=\operatorname{sgn} \varrho(s)$.

For $r_{1}=1$, we prove that

$$
\left|\int_{b_{1}}^{b_{2}} \varrho(s) \chi^{n}(s)\right| \leq \max _{s \in\left[b_{1}, b_{2}\right]}|\varrho(s)|\left(\int_{b_{1}}^{b_{2}}\left|\chi^{(n)}(s)\right| d s\right)
$$

is the best possible inequality. Let $|\varrho(s)|$ attain its maximum at $k_{0} \in\left[b_{1}, b_{2}\right]$. First, we suppose that $\varrho\left(k_{0}\right)>0$. Taking $\epsilon$ to be small enough, we define $\chi_{\epsilon}(s)$ by

$$
\chi_{\epsilon}(s):= \begin{cases}0, & b_{1} \leq s \leq k_{0}, \\ \frac{1}{\epsilon n !}\left(s-k_{0}\right)^{n}, & k_{0} \leq s \leq k_{0}+\epsilon, \\ \frac{1}{n !}\left(s-k_{0}\right)^{n-1}, & k_{0}+\epsilon \leq s \leq b_{2} .\end{cases}
$$

Then, for $\epsilon$ small enough,

$$
\left|\int_{b_{1}}^{b_{2}} \varrho(s) \chi^{(n)}(s)\right|=\left|\int_{k_{0}}^{k_{0}+\epsilon} \varrho(s) \frac{1}{\epsilon} d s\right|=\frac{1}{\epsilon} \int_{k_{0}}^{k_{0}+\epsilon} \varrho(s) d s .
$$

By inequality (64), we have

$$
\frac{1}{\epsilon} \int_{k_{0}}^{k_{0}+\epsilon} \varrho(s) d s \leq \varrho\left(k_{0}\right) \int_{k_{0}}^{k_{0}+\epsilon} \frac{1}{\epsilon} d s=\varrho\left(k_{0}\right)
$$

Since

$$
\lim _{\epsilon \rightarrow 0} \frac{1}{\epsilon} \int_{k_{0}}^{k_{0}+\epsilon} \varrho(s) d s=\varrho\left(k_{0}\right)
$$

the statement follows. 
If $\varrho\left(k_{0}\right)<0$, then $\chi_{\epsilon}(s)$ is

$$
\chi_{\epsilon}(s):= \begin{cases}\frac{1}{n !}\left(s-k_{0}-\epsilon\right)^{n-1}, & b_{1} \leq s \leq k_{0} \\ -\frac{1}{\epsilon n !}\left(s-k_{0}-\epsilon\right)^{n}, & k_{0} \leq s \leq k_{0}+\epsilon \\ 0, & k_{0}+\epsilon \leq s \leq b_{2}\end{cases}
$$

and the remaining proof is identical as above.

(ii) is the same as (i).

Remark 7 By analogy to Sects. 6 and 7 of Aras-Gazič et al. [22], the n-exponential convexity, exponential convexity, and applications to Stolarsky-type means can be established for the functionals defined as the difference between the R.H.S and the L.H.S of generalized inequalities (22) and (33), where $U$ is defined in (23) and (34), respectively.

\section{Acknowledgements}

The writers wish to thank the mysterious refs in advance for their extremely cautious perusing of the original copy and productive remarks and recommendations.

\section{Funding}

This research did not receive any specific grant from funding agencies in the public, commercial, or not-for-profit sectors.

Availability of data and materials

Information sharing isn't appropriate to this paper as no datasets were produced or analyzed during the present study.

\section{Competing interests}

The authors declare that they have no competing interests.

\section{Authors' contributions}

All authors jointly worked on the results and they read and approved the final manuscript.

\section{Author details}

${ }^{1}$ Department of Mathematics and Statistics, The University of Lahore (Sargodha Campus), Sargodha, Pakistan. ${ }^{2}$ Department of Mathematics, Abbottabad University of Science and Technology, Havlian, Abbottabad, Pakistan. ${ }^{3}$ RUDN University, Moscow, Russia.

\section{Publisher's Note}

Springer Nature remains neutral with regard to jurisdictional claims in published maps and institutional affiliations.

Received: 10 December 2020 Accepted: 21 May 2021 Published online: 05 June 2021

\section{References}

1. Aljinovic, A.A., Pečarić, J.: On some Ostrowski type inequalities via Montgomery identity and Taylor's formula. Tamkang J. Math. 36(3), 199-218 (2005)

2. Aljinovic, A.A., Pečarić, J., Vukelic, A.: On some Ostrowski type inequalities via Montgomery identity and Taylor's formula II. Tamkang J. Math. 36(4), 279-301 (2005)

3. Ammi, M.R.S., Ferreira, R.A.C., Torres, D.F.M.: Diamond- $\alpha$ Jensen's inequality on time scales. J. Inequal. Appl. 2008, Article number: 576876 (2008)

4. Anwar, M., Bibi, R., Bohner, M., Pečarić, J.: Integral inequalities on time scales via the theory of isotonic linear functionals. Abstr. Appl. Anal. 2011, Article ID 483595 (2011). https://doi.org/10.1155/2011/483595

5. Bibi, R., Nosheen, A., Pečarić, J.: Generalization of Jensen-type linear functional on time scales via Lidstone polynomial. Cogent Math. Stat. (2017). https://doi.org/10.1080/23311835.2017.1330670

6. Bohner, M.: Calculus of variations on time scales. Dyn. Syst. Appl. 13(3-4), 339-349 (2004)

7. Bohner, M., Matthews, T.: Ostrowski inequalities on time scales. J. Inequal. Pure Appl. Math. 9(1), 8 (2008)

8. Bohner, M., Peterson, A.: Dynamic Equations on Time Scales. Birkhäuser Boston, Boston (2001)

9. Bohner, M., Peterson, A.: Advances in Dynamic Equations on Time Scales. Birkhäuser Boston, Boston (2003)

10. Cerone, P., Dragomir, S.S.: Some new Ostrowski-type bounds for the Cebyšev functional and applications. J. Math. Inequal. 8(1), 159-170 (2014). https://doi.org/10.7153/jmi-08-10

11. da Cruz, A.M.B., Martins, N., Torres, D.F.: Symmetric differentiation on time scales. Appl. Math. Lett. 26(2), 264-269 (2013)

12. da Cruz, A.M.B., Martins, N., Torres, D.F.: The diamond integral on time scales. Bull. Malays. Math. Sci. Soc. 38(4), $1453-1462(2015)$

13. De Boor, C., De Boor, C.: A Practical Guide to Splines, vol. 27, p. 325. Springer, New York (1978) 
14. Hilger, S.: Analysis on measure chains-a unified approach to continuous and discrete calculus. Results Math. 18(1-2), 18-56 (1990). https://doi.org/10.1007/BF03323153

15. Jensen, J.L.W.V.: Sur les fonctions convexes et les inégalités entre les valeurs moyennes. Acta Math. 30, 175-193 (1906)

16. Mitrinović, D.S.: Analytic Inequalities. Springer, New York (1970)

17. Mitrinovic, D.S., Pečarić, J., Fink, A.M.: Inequalities Involving Functions and Their Integrals and Derivatives, vol. 53. Springer, Berlin (1991). https://doi.org/10.1007/978-94-011-3562-7

18. Özkan, U., Sarikaya, M.Z., Yildirim, H.: Extensions of certain integral inequalities on time scales. Appl. Math. Lett. 21(10), 993-1000 (2008). https://doi.org/10.1016/j.aml.2007.06.008

19. Peajcariaac, J.E., Tong, Y.L.: Convex Functions, Partial Orderings, and Statistical Applications. Academic Press, San Diego (1992)

20. Sarikaya, M.Z., Aktan, N., Yildirim, H.: On weighted Čebyšev-Grüss type inequalities on time scales. J. Math. Inequal. 2(2), 185-195 (2008)

21. Sheng, Q., Fadag, M., Henderson, J., Davis, J.M.: An exploration of combined dynamic derivatives on time scales and their applications. Nonlinear Anal., Real World Appl. 7(3), 395-413 (2006)

22. Vukelič, A., Aras-Gazič, G., Pecarič, J.: Generalization of Jensen's and Jensen-Steffensen's inequalities and their converses by Lidstone's polynomial and majorization theorem. J. Numer. Anal. Approx. Theory 46(1), 6-24 (2017)

\section{Submit your manuscript to a SpringerOpen ${ }^{\circ}$ journal and benefit from:}

- Convenient online submission

Rigorous peer review

- Open access: articles freely available online

- High visibility within the field

- Retaining the copyright to your article

Submit your next manuscript at $\mathbf{s p r i n g e r o p e n . c o m ~}$ 